\title{
福岛核事故泄漏的大气放射性核素监测及其对 西安地区的影响
}

刘龙波 ${ }^{(1 *}$, 武山 ${ }^{(1)}$, 曹军骥 ${ }^{(2)}$, 解峰 ${ }^{(1)}$, 师全林 ${ }^{(1)}$, 张昌云 ${ }^{(1)}$, 唐寒冰 ${ }^{(1)}$, 何小兵 ${ }^{(1)}$, 张蓉 ${ }^{(2)}$, 陈莉云 ${ }^{1}$, 韦冠一 ${ }^{(1)}$, 张志弘 ${ }^{(1)}$, 张佳媚 ${ }^{1}$, 党海军 ${ }^{\mathbb{1}}$

(1) 西北核技术研究所, 西安 710024;

(2) 中国科学院地球环境研究所, 西安 710075

* 联系人, E-mail: lomboliu@live.cn

2012-05-16 收稿, 2012-09-04 接受

摘要 2011 年 3 月日本福岛核电站受海啸冲击后发生了严重核事故, 并造成放射性核素的大 量泄漏. 事故发生后, 在西安开展了 3 个月的大气放射性核素监测, 获得了 ${ }^{131} \mathrm{I},{ }^{134} \mathrm{Cs},{ }^{137} \mathrm{Cs}$ 和 ${ }^{133} \mathrm{Xe}$ 等核素的浓度数据, 并结合切尔诺贝利核事故后西安的监测数据及其他国家和地区的监 测数据对本次监测结果进行了讨论, 分析了泄漏核素到达西安的可能路径. 从传输路径分析 来看, 福岛泄漏核素的扩散主要受西风影响, 但在太平洋局部出现的反气旋和源项的连续释

关键词

福岛核事故 放射性核素 大气辐射监测 传输路径 放特点, 使泄漏核素到达中国的大气传输路径比较复杂.

2011 年 3 4 月的日本福岛核事故是继 1986 年切 尔诺贝利核事故后的又一次具有全球重要影响的核 事故. 3 月 11 日, 9 级地震及其引发的海啸损坏了福岛 核电站的反应堆的冷却系统, 致使堆内温度失控. 3 月 12 15 日, 堆内产生的大量氢气排放到厂房内, 1 4 号反应堆相继发生爆炸, 导致大量的放射性核素直 接释放到大气中. 4 月 12 日, 日本政府宣布福岛核泄 漏的事故等级(The International Nuclear and Radiological Event Scale, INES) 为 7 级, 与切尔诺贝利核事 故相同.

福岛核事故发生后, 日本本土大气中随即探测 到放射性核素，美国 ${ }^{[1]}$ 和加拿大 ${ }^{[2,3]}$ 等北美国家、希

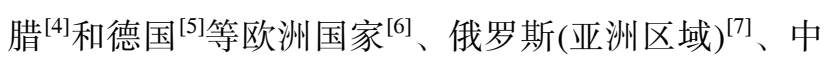
国、韩国 ${ }^{[8]}$ 以及越南 ${ }^{[9]}$ 等亚洲国家先后开始密切关注 释放到大气中的放射性核素的扩散情况。气体裂变 产物放射性氙同位素尤其是 ${ }^{133} \mathrm{Xe}$ 是核泄漏的重要指
示核素，但由于技术和设备的限制，只有部分地区对 其进行了监测 ${ }^{[10]} .{ }^{131} \mathrm{I},{ }^{134} \mathrm{Cs}$ 和 ${ }^{137} \mathrm{Cs}$ 等核事故特征核 素因其较易监测而且对人体有直接的健康影响, 是 监测的主要对象. 自 3 月 15 日开始, 上述各国均陆续 在当地大气中监测到超过本底的 ${ }^{131} \mathrm{I}$. 从放射性核素 出现的峰值时间来看 ${ }^{[1,2,3,9]}$, 这些放射性核素主要随 着西风带环流传过太平洋向北美洲传输, 而后到达 欧洲、亚洲. 除了各国的监测体系外，《全面禁止核 试验条约》组织 (Comprehensive Nuclear-Test-Ban Treaty Organization, CTBTO)筹委会在全球建立的国 际监测系统(International Monitoring System, IMS) ${ }^{[11]}$ 中, 有 63 个放射性气溶胶监测台站和 27 个惰性气体 氙监测台站也进行了监测, 结果显示, 福岛核事故后 两周内, 北半球所有台站和南半球的 3 个台站都监测 到了高于本底的放射性核素 ${ }^{1)}$. 从监测数据来看, 大部 分地区的 ${ }^{131}$ I 浓度在 3 月底至 4 月初达到最大, 从 4 月

1) Stoehlker U, Nikkinen M, Gheddou A. Detection of radionuclides emitted during the Fukushima nuclear accident with the CTBT radionuclide network. 2011 Monitoring Research Review: Ground-Based Nuclear Explosion Monitoring Technologies. 2011

引用格式: 刘龙波, 武山, 曹军骐, 等. 福岛核事故泄漏的大气放射性核素监测及其对西安地区的影响. 科学通报, 2013, 58: 372-378

英文版见: Liu L B, Wu S, Cao J J, et al. Monitoring of atmospheric radionuclides from the Fukushima nuclear accident and assessing their impact on Xi' an, China. Chin Sci Bull, 2013, 58, doi: 10.1007/s11434-012-5521-4 
中旬开始逐渐减小至本底水平. 在各地监测的同时, 日本 Chino 等人 ${ }^{[12]}$ 和瑞典的 Stohl 等人 ${ }^{[13]}$ 运用大气输 运模拟计算, 研究了释放核素的总量和释放速率随 时间的变化过程.

国家环保部于 2011 年 3 月 26 日首次发布消息， 称在黑龙江省多地监测到了放射性核素 ${ }^{131} \mathrm{I}$, 其环境 辐射水平仍处于本底范围. 随后连续数日发布了各 地对 ${ }^{131} \mathrm{I}$ 的监测结果, 并连续每日发布全国省会城市 和部分地级市辐射环境实时空气吸收剂量率监测值. 直至 5 月 21 日, 监测结果表明, 我国上述主要城市的 环境辐射水平一直处于本底水平范围内(http://www. mep.gov.cn/gkml/hbb/qt/201105/t20110521_210981.htm). 研究工作方面，乔方利等人 ${ }^{[14]}$ 报道了对泄漏核素大 气扩散的预测, 王海洋等人 ${ }^{[15]}$ 提出了泄漏源项的估计, 张文杰等人 ${ }^{2}$ 和周程等人 ${ }^{[16]}$ 分别报道了在北京和南京 对气溶胶中放射性核素的监测结果. 本文汇总了在 西安对大气气溶胶和惰性气体氙的核素监测结果, 并对监测结果进行对比分析, 探讨其输送路径.

\section{1 监测过程}

福岛核事故发生后, 从 3 月 23 日到 6 月 30 日我 们在西安市东郊(距市中心 $20 \mathrm{~km}$ )开展了取样监测. 3 月 23 日至 4 月 15 日为连续监测, 以后由于相关核素 浓度显著降低, 且福岛核电站再无爆炸或起火等事 件发生, 监测频率改为每周一次. 监测方法参考 IMS 的监测规范 ${ }^{[17,18]}$

\section{1 大气气溶胶中放射性核素监测}

使用自行研制的气溶胶采样器进行取样, 采样 流量 $450 \mathrm{~m}^{3} / \mathrm{h}, 24$ 小时取样空气体积大于 $10000 \mathrm{~m}^{3}$. 采样器的基本原理及性能测试参见文献 [19]. 使用 $\mathrm{NF}$ 聚丙烯纤维滤材取样, 对 $0.2 \mu \mathrm{m}$ 气溶胶的捕集效 率为 $90 \%{ }^{[20]}$. 取样后滤材放人专用模具中, 用自动压 片机压成圆片，用 HPGe $\gamma$ 谱仪(相对效率 60\%，能量 分辨率为 $1.94 \mathrm{keV}(1332.5 \mathrm{keV})$ )进行相关放射性核素 的测量, 重点测量易挥发、半衰期适中的裂变产物核 素, 如 ${ }^{131} \mathrm{I},{ }^{132} \mathrm{Te},{ }^{132} \mathrm{I}$ 和 ${ }^{137} \mathrm{Cs}$ 等.

相关核素的活度 $A$ 由式(1) ${ }^{[21]}$ 计算得到:

$$
A=\frac{N}{t_{1} \varepsilon_{i} p_{i} K_{\mathrm{c}}},
$$

式中, $N$ 为由 GammaVision 解谱软件通过计数相加法 自动扣除本底后得出的净峰面积; $t_{1}$ 为样品测量活时 间, $\mathrm{s} ; \varepsilon_{i}$ 为所测 $\gamma$ 能峰的探测效率; $p_{i}$ 为所测 $\gamma$ 能峰的 分支比; $K_{\mathrm{c}}$ 为测量期间的衰变校正因子，有

$$
K_{\mathrm{c}}=\frac{1-\exp \left(-\lambda t_{\mathrm{m}}\right)}{\lambda t_{\mathrm{m}}},
$$

式中, $t_{\mathrm{m}}$ 为样品测量实时间, $\mathrm{s} ; \lambda$ 为该放射性核素的衰 变常数, $\mathrm{s}^{-1}$.

相关核素在大气中的浓度为

$$
C=\frac{A}{V \xi K_{\mathrm{w}} K_{\mathrm{s}}},
$$

式中, $C$ 为相关核素的在大气中的活度浓度, $\mathrm{Bq} / \mathrm{m}^{3} ; V$ 为标准温度和压力下的取样体积, $\mathrm{m}^{3}$; $\xi$ 为取样效率, 气溶胶取样一般为 $1 ; K_{\mathrm{s}}$ 为取样期间的衰变校正因子, 有

$$
K_{\mathrm{s}}=\frac{1-\exp \left(-\lambda t_{\mathrm{s}}\right)}{\lambda t_{\mathrm{s}}},
$$

式中, $t_{\mathrm{s}}$ 为取样持续时间, $\mathrm{s}$;

$K_{\mathrm{w}}$ 为取样结束到开始测量期间的衰变校正因子, 有

$$
K_{\mathrm{w}}=\exp \left(-\lambda t_{\mathrm{w}}\right),
$$

式中, $t_{\mathrm{w}}$ 为从取样结束到开始测量的时间间隔, $\mathrm{s}$.

当样品中待测核素的活度小于最小可探测活度 (Minimum Detectable Activity, MDA)时，以小于最小 可探测浓度(Minimum Detectable Concentration, MDC) 作为浓度测量结果. MDA 由式(6) ${ }^{[18]}$ 计算得到

$$
\mathrm{MDA}=\frac{L_{d}}{t_{1} \varepsilon_{i} p_{i} K_{c}}=\frac{2.71+4.65 \sqrt{\mu_{\mathrm{B}}}}{t_{1} \varepsilon_{i} p_{i} K_{c}},
$$

式中, $\mu_{\mathrm{B}}$ 为在测量的能量范围内本底测量标准偏差, 有 $\mu_{\mathrm{B}}=\sum \mathrm{ROI}, \mathrm{ROI}$ 为能量范围内 $\pm 1.25 \mathrm{FWHM}( \pm 3 \sigma)$ 的 区域。

$\mathrm{MDC}$ 的计算 ${ }^{[18]}$ 为将式(6)得到的 MDA 作为活度 $A$ 代人式(3)即可. 所测主要核素的半衰期和 $\gamma$ 射线能 量见表 1. 根据 IMS 台站的监测规范, 气溶胶样品取 样后放置 $24 \mathrm{~h}$ 才开始测量, 以使天然氡子体充分衰 变, 减小对其他核素的测量干扰。当待测核素浓度

2) 张文杰，白志鹏，孟凡，等. 日本地震引发的放射性核素对我国北方区域影响初步分析. 第 18 届中国大气环境科学与技术大会 2011. 杭州 
表 1 主要核素半衰期和 $\gamma$ 射线能量值

\begin{tabular}{lccccccc}
\hline & ${ }^{131} \mathrm{I}$ & ${ }^{132} \mathrm{Te}$ & ${ }^{132} \mathrm{I}$ & ${ }^{137} \mathrm{Cs}$ & ${ }^{134} \mathrm{Cs}$ & ${ }^{7} \mathrm{Be}$ & ${ }^{133} \mathrm{Xe}$ \\
\hline 半衰期 & $8.02 \mathrm{~d}$ & $76.8 \mathrm{~h}$ & $2.29 \mathrm{~h}$ & $30.1 \mathrm{a}$ & $2.07 \mathrm{a}$ & $53.3 \mathrm{~d}$ & $5.24 \mathrm{~d}$ \\
衰变常数 $(\mathrm{d})$ & 0.0864 & 0.217 & 7.28 & $6.32 \times 10^{-5}$ & $9.20 \times 10^{-4}$ & 0.013 & 0.132 \\
$\quad \gamma$ 射线能量 $(\mathrm{keV})$ & 364.5 & 228.2 & 667.7 & 661.6 & 604.7 & 477.6 & 81 \\
\hline
\end{tabular}

较高时, 可以适当缩短放置时间. 根据样品中待测核 素活度情况, 测量时间为 2 24 h. 各核素的活度浓度 均按各自的衰变常数推算到取样开始时刻. 在取样、 放置和测量均为 $24 \mathrm{~h}$, 取样体积 $10000 \mathrm{~m}^{3}$ 的条件下, ${ }^{131} \mathrm{I}$ 和 ${ }^{137} \mathrm{Cs}$ 的 $\mathrm{MDC}$ 分别为 5.4 和 $4.3 \mu \mathrm{Bq} / \mathrm{m}^{3}$.

在气溶胶取样的质量控制方面, 可以使用 ${ }^{7} \mathrm{Be}$. 大气中 ${ }^{7} \mathrm{Be}$ 主要产生于平流层和对流层顶, 以气溶胶 的形式存在于大气中. 由于其浓度变化主要受当地 太阳辐射以及气溶胶沉降过程的影响, 因此相对稳 定, 在大气放射性气溶胶的监测中常作为系统运行 状态的指示性核素, 在某一监测地点的长期监测的 质量控制中意义重大. 本次监测到 ${ }^{7} \mathrm{Be}$ 的浓度在 $3.81 \sim 12.24 \mathrm{mBq} / \mathrm{m}^{3}$, 与常印忠等人 ${ }^{[22]} 2002$ 年监测的 西安同期的 2 12 mBq/m ${ }^{3}$ 相同. 这说明本次监测的系 统状态正常.

\section{2 大气放射性氞监测}

使用自行研制的大气氙取样器进行取样: 空气 压缩机抽取大气, 初步除去水分和二氧化碳等杂质 后, 用活性炭吸附柱低温吸附氙, 加热 $\left(200^{\circ} \mathrm{C}\right)$ 脱附, 再经小吸附柱进一步分离除去氡等杂质, 纯化浓缩 氙. 24 小时获取的总氙量不小于 $4 \mathrm{~mL}$, 有效空气取 样体积约 $50 \mathrm{~m}^{3}$. 获取的氙样品先用低能平面 HPGe $\gamma$ 谱仪系统进行放射性氙同位素的测量, 由式(1)测得 某种放射性氙同位素的活度，计算公式同式(1). 谱 仪系统对于 ${ }^{133} \mathrm{Xe} 81 \mathrm{keV} \gamma$ 射线的探测效率为 $50 \%$. 然后用四极杆气体质谱计 QMS 测量样品中的总氙浓 度 ${ }^{[23]}$, 根据样品中氙的浓度和大气中氙的稳定同位 素的浓度比值计算取样效率．对大气中放射性氙同 位素的活度浓度的计算公式原理与式(3)相同, 但习 惯上以样品气体积, 而不是取样空气体积作为取样 体积的基础. 这样, 对大气中某种放射性氙同位素的 活度浓度的计算见式(7):

$$
C_{\mathrm{X}}=\frac{A C_{0} P_{0} T}{V_{\mathrm{s}} C_{\mathrm{s}} P T_{0} K_{\mathrm{w}} K_{\mathrm{s}}},
$$

式中, $C_{\mathrm{X}}$ 为大气中放射性氙同位素的活度浓度, $\mathrm{Bq} / \mathrm{m}^{3} ; A$ 为样品中放射性氙同位素的活度, $\mathrm{Bq} ; C_{\mathrm{s}}$ 为 样品气中氙浓度, $10^{-6} \mathrm{v} / \mathrm{v} ; V_{\mathrm{s}}$ 为样品气体体积, $\mathrm{m}^{3} ; P$ 为样品气体压力, $\mathrm{kPa} ; T$ 为样品气体温度, $\mathrm{K} ; P_{0}$ 为标 准状况大气压, $101.325 \mathrm{kPa} ; T_{0}$ 为标准状况温度, $273.15 \mathrm{~K} ; C_{0}$ 为标准状况大气中氙浓度, $0.087 \times 10^{-6} \mathrm{v} / \mathrm{v}$.

气体核素的 MDA 的计算同式(6), MDC 的计算 需要结合式(6)和(7), 原理与气溶胶样品中核素的 MDC 计算相同. 在取样 $24 \mathrm{~h}$, 制源 $3 \mathrm{~h}$, 测量 $24 \mathrm{~h}$ 的 情况下, 系统对 ${ }^{133} \mathrm{Xe}$ 的 MDC 为 $\sim 0.2 \mathrm{mBq} / \mathrm{m}^{3}$.

\section{2 监测结果与讨论}

\section{1 核素浓度及变化}

(i )气溶胶形态的放射性核素。本研究从 3 月 23 日开始在西安开展监测, 即在气溶胶样品中监测 到了浓度显著高于 $\mathrm{MDC}$ 的 ${ }^{131} \mathrm{I}$. 后续的监测还发现 了 ${ }^{137} \mathrm{Cs},{ }^{134} \mathrm{Cs},{ }^{132} \mathrm{I}$ 和 ${ }^{132} \mathrm{Te}$ 等核素. 需要说明的是, 上 述核素中, 除了 ${ }^{137} \mathrm{Cs}$ 由于半衰期较长, 过去核事故使 大气中存在一个很小的本底浓度(比如欧洲大气中的 本底浓度在 $0.001 \mathrm{mBq} / \mathrm{m}^{3}$ 以下 $\left.{ }^{[5]}\right)$ 外, 其他核素的半 衰期较短, 在没有新增源项的情况下本底浓度基本 可以忽略. 本次监测到的 ${ }^{131} \mathrm{I},{ }^{137} \mathrm{Cs}$ 和 ${ }^{134} \mathrm{Cs}$ 的活度浓 度随日期的变化见图 1 , 其他核素的浓度则很快衰减 至 MDC 以下. 从图 1 可以看出, ${ }^{131} \mathrm{I}$ 分别在 3 月 25 日 和 4 月 5 日出现峰值, 浓度分别为 3.92 和 $1.66 \mathrm{mBq} / \mathrm{m}^{3}$; 4 月 6 日以后, ${ }^{131} \mathrm{I}$ 呈现明显的指数下降过程, 至 4 月 27 日接近 MDC.

所有放射性核素本身有半衰期, 如果其在大气 中稳定存在, 没有新的来源, 则其浓度衰减也会呈现 指数关系, 浓度衰减系数应当为其衰变常数 (见表 1). 若以如式(8)所示公式对 4 月 6 日以后 ${ }^{131} \mathrm{I}$ 的活度浓度 的数据拟合, 得到 ${ }^{131} \mathrm{I}$ 浓度的浓度衰减系数 $k\left({ }^{131} \mathrm{I}\right)$ 为 $0.227 \mathrm{~d}^{-1}, R^{2}$ 为 0.9657 (见图 1).

$$
C_{t}(\mathrm{Y})=C_{0}(\mathrm{Y}) \exp [-k(\mathrm{Y}) t] \text {, }
$$




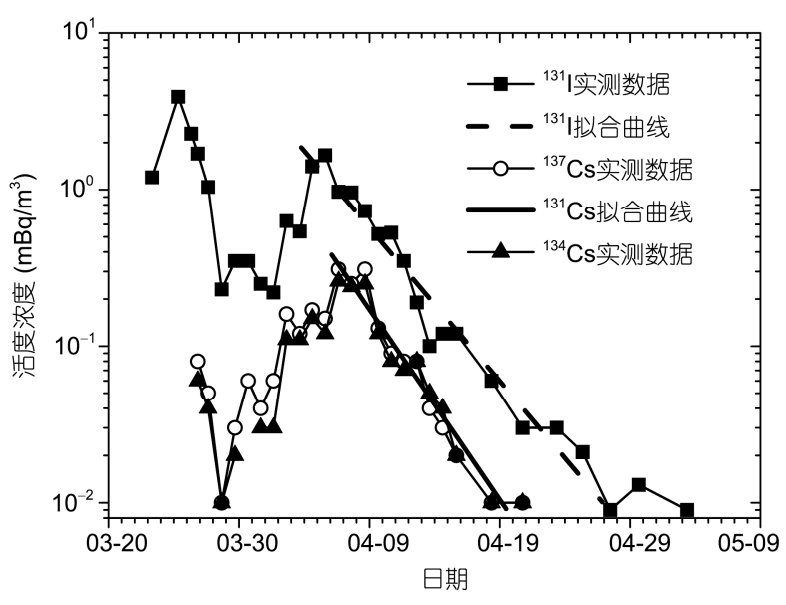

图 1 大气气溶胶中主要核素的活度浓度变化

式中, $C_{0}(\mathrm{Y})$ 和 $C_{t}(\mathrm{Y})$ 分别表示核素 $\mathrm{Y}$ 在 0 时刻和 $t$ 时 刻的浓度, $\mathrm{Bq} / \mathrm{m}^{3} ; k(\mathrm{Y})$ 为核素 $\mathrm{Y}$ 的浓度衰减系数, $\mathrm{d}^{-1}$. $k\left({ }^{131} \mathrm{I}\right) 0.227 \mathrm{~d}^{-1}$ 显著大于 ${ }^{131} \mathrm{I}$ 的衰变常数 0.0864 $\mathrm{d}^{-1}$, 说明大气中 ${ }^{131} \mathrm{I}$ 浓度的衰减除了自身衰变外, 还 有沉降或大气扩散稀释作用. 如果将 ${ }^{131} \mathrm{I}$ 浓度全部校 正到 4 月 6 日, 即排除自身衰变的影响, 则大气中浓 度的衰减系数为 $0.141 \mathrm{~d}^{-1}$.

本研究观测到的 ${ }^{134} \mathrm{Cs}$ 和 ${ }^{137} \mathrm{Cs}$ 的浓度变化趋势与 ${ }^{131} \mathrm{I}$ 相似, 最大值分别为 0.26 和 $0.31 \mathrm{mBq} / \mathrm{m}^{3}$, 出现在 4 月 6 日. ${ }^{134} \mathrm{Cs}$ 和 ${ }^{137} \mathrm{Cs}$ 的浓度变化几乎完全一致, 而 其最大值比 ${ }^{131} \mathrm{I}$ 滞后 1 天, 可能是由于 ${ }^{131} \mathrm{I}$ 部分以气 态存在, 大气中的扩散速度较快的缘故. 同样对 4 月 6 日以后 ${ }^{137} \mathrm{Cs}$ 浓度的数据拟合可以得到, 其浓度衰 减系数 $k\left({ }^{137} \mathrm{Cs}\right)$ 为 $0.251 \mathrm{~d}^{-1}\left(R^{2}\right.$ 为 0.9061$)$, 远大于其自 身的衰变常数 $6.32 \times 10^{-5} \mathrm{~d}^{-1}$, 也大于排除了衰变影响 的 ${ }^{131} \mathrm{I}$ 的浓度衰减系数 $0.141 \mathrm{~d}^{-1}$. 这说明在西安监测 到的放射性核素的浓度变化中, ${ }^{137} \mathrm{Cs}$ 比 ${ }^{131} \mathrm{I}$ 的浓度衰 减更快, 这可能是由于碘作为挥发性核素, 即使沉降 也容易再蒸发返回大气, 也可能是铯和碘所在颗粒 粒径不同, 因而在大气中的沉降速度不同. 详细地分 析这些核素的沉降机理还需要沉积样品的数据.

在核事件性质判断方面, ${ }^{134} \mathrm{Cs} /{ }^{137} \mathrm{Cs}$ 可以作为事 件性质的一个判据 ${ }^{[18]}$. 在反应堆中, ${ }^{133} \mathrm{Xe}$ 衰变成稳定 核素 ${ }^{133} \mathrm{Cs}$, 而 ${ }^{133} \mathrm{Cs}$ 可以被活化成 ${ }^{134} \mathrm{Cs}$. 核反应过程为:

$$
{ }^{133} \mathrm{Xe} \stackrel{\beta}{\longrightarrow}{ }^{133} \mathrm{Cs} \stackrel{n, \gamma}{\longrightarrow}{ }^{134} \mathrm{Cs}
$$

反应堆中的活化产物 ${ }^{134} \mathrm{Cs}$ 会不断累积, 使其活 度与裂变产物 ${ }^{137} \mathrm{Cs}$ 的活度相当, 而核爆炸产生的 ${ }^{134} \mathrm{Cs}$ 非常少, 因此通常认为 ${ }^{134} \mathrm{Cs}$ 可以作为核反应堆
事故的特征核素. 1986 年切尔诺贝利事故后监测的 ${ }^{134} \mathrm{Cs} /{ }^{137} \mathrm{Cs}$ 为 0.58 , 本次监测的平均值为 0.88 , 说明 ${ }^{134} \mathrm{Cs}$ 和 ${ }^{137} \mathrm{Cs}$ 的浓度相当, 其源项是比较典型的反应 堆事故.

(ii) 气态放射性核素. 核事故释放的气体核素 一般有 $\mathrm{Xe}$ 和 $\mathrm{Kr}$ 的放射性同位素, 其中 ${ }^{133} \mathrm{Xe},{ }^{131} \mathrm{Xe}$, ${ }^{131 \mathrm{~m}} \mathrm{Xe}$ 和 ${ }^{135} \mathrm{Xe}$ 等放射性氙同位素的半衰期均较短, 可以反映近期大气中放射性核素情况, 是气体核素 监测的主要对象. 图 2 是 3 月 27 日 6月 10 日连续监 测的 ${ }^{133} \mathrm{Xe}$ 的浓度变化曲线, 监测中发现的最大值是 3 月 27 日的 $5.5 \mathrm{~Bq} / \mathrm{m}^{3}$, 其后于 3 月 30 日和 4 月 5 日 两次出现极大值 1.0 和 $0.88 \mathrm{~Bq} / \mathrm{m}^{3}$, 其中 4 月 5 日的 峰值出现时间与 ${ }^{131} \mathrm{I}$ 相同. 4 月 5 日以后 ${ }^{133} \mathrm{Xe}$ 的浓度 指数下降, 拟合得到浓度衰减系数 $k\left({ }^{133} \mathrm{Xe}\right)$ 为 0.130 $\mathrm{d}^{-1}$, 与其衰变常数 $0.132 \mathrm{~d}^{-1}$ 吻合, 说明 ${ }^{133} \mathrm{Xe}$ 浓度的 下降主要是自身衰变引起的. 很可能是此后再无大 量的 ${ }^{133} \mathrm{Xe}$ 释放到大气, 同时在北半球大气中的 ${ }^{133} \mathrm{Xe}$ 已经均匀, 进一步的扩散引起的稀释效应不明显.

从对人类安全的影响来看, 上述核素的影响差 别较大: ${ }^{131} \mathrm{I}$ 容易沉积并转移到人体中, 对人类健康 的影响较大, 但其半衰期较短, 因此是事故后短期监 测的主要对象; ${ }^{137} \mathrm{Cs}$ 的半衰期较长, 沉积到地面后会 造成地面的长期污染, 并可能通过再悬浮重新进人 大气环境, 因此是需要长期进行环境辐射监测的核 素. ${ }^{133} \mathrm{Xe}$ 为惰性气体, 吸人后在体内的停留时间较短, 对人类健康的直接影响相对较小, 但其气体性质使 其容易被释放, 对事故的发生比较灵敏, 因此在事故 监测中具有重要意义. 从本次在西安对福岛核事故 引起的大气放射性核素的监测结果来看, 根据环境

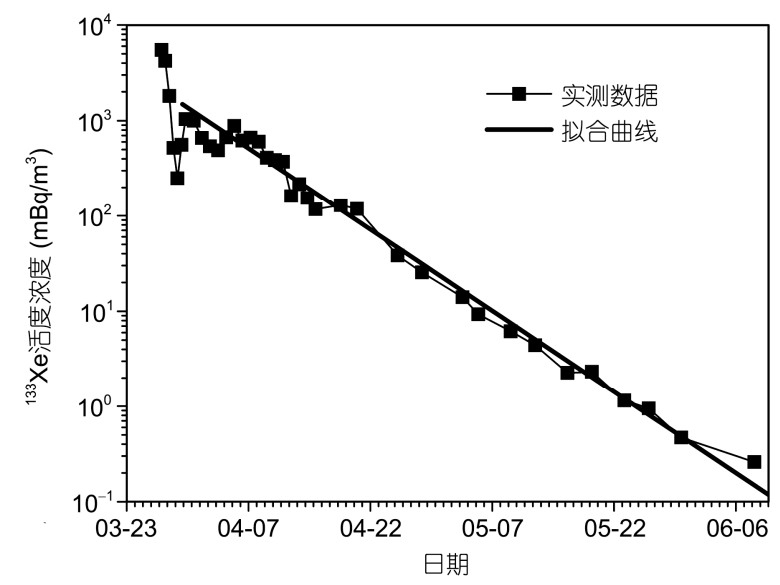

图 2 大气中 ${ }^{133} \mathrm{Xe}$ 的活度浓度变化 
辐射的国家标准 ${ }^{[24]}$, 这些大气放射性核素的浓度造 成的附加年最大吸收有效剂量远远低于自然辐射本 底造成的公众年有效剂量, 与国家环保部发布的公 告结论一致, 对西安的环境辐射水平没有显著影响.

\section{2 与切尔诺贝利事故的比较分析}

1986 年的切尔诺贝利事故向大气中释放了大量 放射性核素 (见表 2), 对全世界的环境放射性水平产 生了重要的影响, 有关的研究工作迄今仍然在继续 进行. 此次日本福岛核电站的核泄漏, 从估算的放射 性核素泄漏量(表2)来看, 主要核素的泄漏量确实与 切尔诺贝利事故相当. 将本次在西安监测到的大气 中放射性核素与切尔诺贝利事故后在西安 ${ }^{[26]}$ 监测到 的结果对比发现: 在核素种类方面, 本次监测到的核 素种类较少, 只有 ${ }^{131} \mathrm{I},{ }^{134} \mathrm{Cs}$ 和 ${ }^{137} \mathrm{Cs}$ 等易挥发性核素, 而切尔诺贝利事故后还监测到了 ${ }^{143} \mathrm{Ce},{ }^{140} \mathrm{Ba}$ 和 ${ }^{140} \mathrm{La}$ 等核素; 在放射性核素浓度水平上, 本次监测到的浓 度也比 1986年的低 10 100 倍. 这些特点在其他区域 的监测中也得以体现, 比如本次欧洲监测到的浓度 比切尔诺贝利事故期间低 100 1000 倍 ${ }^{[6]}$.

以上的差别可能是由于事故的性质以及释放过 程的差异造成的. 切尔诺贝利事故为反应堆爆炸, 后 引发堆芯熔化及石墨起火, 估计 ${ }^{[26]}$ 堆内核材料中, 气 体裂变产物 ${ }^{133} \mathrm{Xe}$ 等 $100 \%$ 泄漏, 易挥发性核素 ${ }^{131} \mathrm{I}$ 有 $50 \%$ 60\%泄漏, 易挥发性核素 ${ }^{134} \mathrm{Cs}$ 和 ${ }^{137} \mathrm{Cs}$ 等有 $20 \% \sim 40 \%$ 泄漏, ${ }^{95} \mathrm{Zr},{ }^{95} \mathrm{Nb},{ }^{144} \mathrm{Ce}$ 等非挥发性裂变产物 核素以及超铀核素有 $3.5 \%$ 泄漏. 福岛核事故主要为 氢气爆炸和乏燃料池起火, 温度较低, 因此以易挥发 性核素为主. 另外, 切尔诺贝利地处欧亚大陆中部, 事故中放射性核素的主要释放时间为 10 天, 此后的 小量释放持续约 40 天, 事故期间风向以东风和北风 为主, 因此前苏联及欧洲大部均受到影响 ${ }^{[26]}$. 而福岛 地处太平洋西海岸, 事故期间以西风为主, 泄漏的核

\section{表 2 福岛核事故与切尔诺贝利核事故向环境中泄漏的} 放射性核素估计量的比较(单位: Bq) ${ }^{\mathbf{a})}$

\begin{tabular}{ccc}
\hline 核素 & 福岛 & 切尔诺贝利 ${ }^{[25]}$ \\
\hline${ }^{131} \mathrm{I}$ & $1.5 \times 10^{17[12]}$ & $1.76 \times 10^{18}$ \\
${ }^{134} \mathrm{Cs}$ & - & $5.4 \times 10^{16}$ \\
${ }^{137} \mathrm{Cs}$ & $1.3 \times 10^{16[12]}$ & $8.5 \times 10^{16}$ \\
${ }^{133} \mathrm{Xe}$ & $3.58 \times 10^{16[13]}$ & $6.5 \times 10^{18}$ \\
\hline
\end{tabular}

a) “-”表示未见报道
素主要向太平洋方向扩散 ${ }^{[13]}$, 因此在大陆腹地实际 监测到的浓度较低.

\section{3 与其他地区的监测结果比较}

据目前的估计 ${ }^{[12,13]}$, 福岛核事故的释放从 3 月 15 日持续到 4 月 5 日以后, 历时 20 余天, ${ }^{131} \mathrm{I}$ 的释放 速率在 $10^{11} \sim 10^{16} \mathrm{~Bq} / \mathrm{h} .3$ 月 $15 \sim 25$ 日左右为持续释放, 26 27 日出现较低值, 3 月 30 31 日出现较大值, 随后 逐渐减小. 福岛核事故发生以后, 世界各地都开展了 对大气放射性核素的监测. 对监测结果按地域划分, 大致有如下几种情况:

(1) 日本. 据 CTBTO 在日本高崎(在福岛西南 方向 $200 \mathrm{~km}$ )的 38 号台站的监测结果 ${ }^{1)}, 3$ 月 12 日开 始测到 ${ }^{134} \mathrm{Cs},{ }^{136} \mathrm{Cs},{ }^{137} \mathrm{Cs},{ }^{131} \mathrm{I},{ }^{132} \mathrm{I},{ }^{133} \mathrm{I},{ }^{132} \mathrm{Te},{ }^{136 \mathrm{~m}} \mathrm{Ba}$ 和 ${ }^{133} \mathrm{Xe}, 3$ 月 16 日 ${ }^{131} \mathrm{I}$ 和 ${ }^{137} \mathrm{Cs}$ 的浓度达到最大, 分 别为 15 和 $5.6 \mathrm{~Bq} / \mathrm{m}^{3}$. ${ }^{133} \mathrm{Xe}$ 的最大浓度超过 $100 \mathrm{~Bq} / \mathrm{m}^{3}$. 时间序列方面, 核素浓度在 3 月出现几次峰值, 4 月 浓度比较稳定, 5 月开始逐渐下降, 到 5 月底, ${ }^{131} \mathrm{I}$ 和 ${ }^{137} \mathrm{Cs}$ 浓度在 $1 \mathrm{mBq} / \mathrm{m}^{3}$ 左右.

(2) 北美. 美国 ${ }^{[1,27]}$ 自 3 月 15 日开始监测到异常, 是日本本土以外较早监测到放射性核素的国家. 核 素种类与日本本土相同, ${ }^{131} \mathrm{I}$ 最大浓度为 $30 \mathrm{mBq} / \mathrm{m}^{3}$, ${ }^{133} \mathrm{Xe}$ 最大浓度为 $17 \mathrm{~Bq} / \mathrm{m}^{3}$. 时间序列方面, 由于距 离日本比较近, 受到核素传输的持续显著影响, 无明 显峰值, 但从 3 月底开始就呈现缓慢下降趋势. 加拿 $大^{[2,3]}$ 情况与美国相似.

(3) 欧洲 ${ }^{[5]}$. 监测到的大气气溶胶放射性核素 种类较少(放射性氙同位素结果尚未见报道), 主要有 ${ }^{131} \mathrm{I},{ }^{134} \mathrm{Cs}$ 和 ${ }^{137} \mathrm{Cs}$, 另有痕量的 ${ }^{132} \mathrm{Te}$ 和 ${ }^{132} \mathrm{I} .{ }^{131} \mathrm{I}$ 的时 间序列显示了两个峰值, 第一个在 3 月 28 30 日陆续 到达欧洲各国，第二个峰值比较集中，在 4 月 3 5 日. 从浓度来看, ${ }^{131} \mathrm{I}$ 的两个峰值浓度均在 $1 \sim 6 \mathrm{mBq} / \mathrm{m}^{3}$.

(4) 亚洲其他国家. 越南 ${ }^{[9]}$ 自 3 月 27 日监测到 ${ }^{131} \mathrm{I},{ }^{134} \mathrm{Cs}$ 和 ${ }^{137} \mathrm{Cs}$. 时间序列方面只出现一个单峰, 4 月 10 日 ${ }^{131} \mathrm{I}$ 和 ${ }^{137} \mathrm{Cs}$ 的峰值分别为 0.93 和 0.036

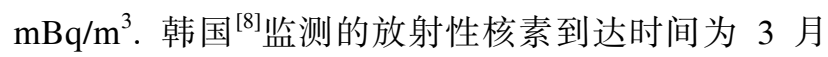
28 日, 且只有一个 ${ }^{131} \mathrm{I}$ 峰值为 $3.12 \mathrm{mBq} / \mathrm{m}^{3}$ (4 月 6 日). 逆向轨迹分析显示, 到达韩国的气团先经过了北京.

与上述地区相比, 此次在西安对特征核素 ${ }^{134} \mathrm{Cs}$, ${ }^{137} \mathrm{Cs},{ }^{131} \mathrm{I}$ 和 ${ }^{133} \mathrm{Xe}$ 都进行了监测. 受传输时间及传输 过程中的沉降、扩散、衰变过程的影响, 核素浓度水 平远低于日本和美国, 而与欧洲的相当. 从放射性核 
素出现的时间及峰值出现时间看, 日本最早监测到 放射性核素, 其次是北美, 然后是亚洲内陆的西安出 现峰值, 最后才是欧洲. 西安 ${ }^{131}$ I 峰值出现的时间(3 月 25 日)早于欧洲 3 4 天，因此到达西安的放射性核 素气团不可能是随西风带经欧洲传来的，那么放射 性核素是以何途径传到西安的呢?

\section{4 核素到达西安的传输路径分析}

福岛事故泄漏核素最早开始于 3 月 12 日，而西 安地处中国中部地区, 位于福岛以西约 $2800 \mathrm{~km}$, 在 3 月 25 日, 即事故发生后 13 天就监测到了 ${ }^{131} \mathrm{I}$ 的第 一个浓度峰值. 福岛核事故泄漏的核素通过何种途 径到达西安, 是对福岛泄漏核素监测的重要问题. 西 班牙 Lozano 等人 ${ }^{[28]}$ 使用 HYSPLIT (the Hybrid Single Particle Lagrangian Integrated Trajectory)模式分析了 福岛泄漏核素云团到达欧洲的传输路径, 但未涉及 对亚洲及中国的影响. 乔方利等人 ${ }^{[14]}$ 用自主研发的 程序对泄漏核素通过大气传输的影响范围进行了预 测，在其模拟计算中假设源项为单位浓度的“一次 性”释放，在其影响区域的表述中使用了“全场浓度 最高浓度 $10 \%$ 的浓度等值线”. 而本文中对区域影响 的界定以实测大气中核素浓度高于 MDC 作为依据, 与乔方利等人的模拟计算结果无法直接比较. 户飞 虹等人 ${ }^{[29]}$ 用自主研发的模式对传输路径进行了分析, 结果表明: 泄漏放射性核素主要向东、南、北 3 个方
向漂移扩散进人太平洋, 其中进人太平洋向北扩散 的一支云团进人俄罗斯后又向西随后向南折回, 流 经我国东北、东部地区; 同时，向南扩散的一支云团 将逐渐变为向西南方向扩散, 越过菲律宾后向西偏 北方向移动, 经我国南海进人海南地区. 若按此途径, 福岛释放的核素有可能从南北两个方向进人我国内 陆地区.

本研究使用 HYSPLIT 分析了核泄漏气团到达西 安前后不同海拔的后向轨迹及福岛核泄漏期间气团 的前向轨迹. 结果表明, 尽管事故期间西安、福岛的 主导风向都受西风带的影响, 但西风带主要控制了 海拔 $3 \mathrm{~km}$ 以上的高空, 而近地面(海拔约 $1.5 \mathrm{~km}$ ) 会 出现短期的反气旋(如图 3 所示), 从而使污染气团未 经过北美和欧洲就直接到达中国内陆，与到达欧洲 各国的时间相当甚至更早. 结合核素到达各地的时 间，可以推测，福岛泄漏核素受西风影响扩散到太平 洋后，可能出现分叉，部分会向北或南运动，进而在 东亚北部和南部向西运动. 这可能是泄漏核素到达 西安和欧洲的时间相当的主要原因.

\section{3 结论与展望}

福岛核事故后，在西安开展了 3 个月的大气放射 性核素监测, 获得了 ${ }^{131} \mathrm{I},{ }^{134} \mathrm{Cs},{ }^{137} \mathrm{Cs}$ 和 ${ }^{133} \mathrm{Xe}$ 等核素 的浓度数据, 从监测结果来看, 这些核素所造成附加 年有效吸收剂量远低于自然辐射本底造成的公众年
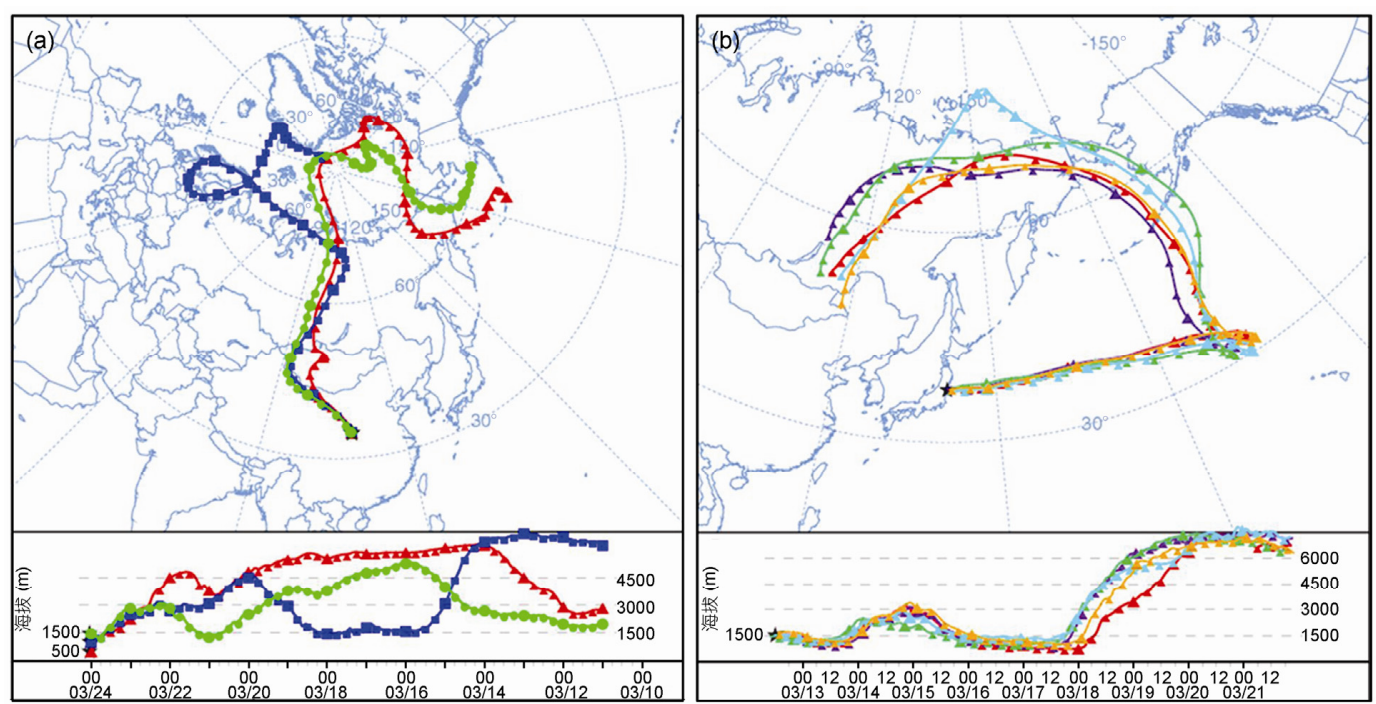

图 3 西安核素污染气团的 HYSPLIT 轨迹图

(a) 终点为西安( 3 月 24 日 0 时 UTC)的背向轨迹图; (b) 起点位于福岛(3 月 12 日 10 14 时 UTC)的前向轨迹图 
吸收剂量，对当地的环境辐射没有显著影响．从传输 路径分析来看, 福岛泄漏核素的扩散主要受西风影 响，但在太平洋局部出现的反气旋和源项的连续释
放特点，使泄漏核素到达中国的大气传输路径变得 比较复杂. 这需要在未来核事故的监测和预警研究 中重点关注.

\section{参考文献}

1 Diazleon J, Jaffe D A, Kaspar J, et al. Arrival time and magnitude of airborne fission products from the Fukushima, Japan, reactor incident as measured in Seattle, WA, USA. J Environ Radioact, 2011, 102: 1032-1038

2 Sinclair L E, Seywerd H C J, Fortin R, et al. Aerial measurement of radioxenon concentration off the west coast of Vancouver Island following the Fukushima reactor accident. J Environ Radioact, 2011, 102: 1018-1023

3 Zhang W, Bean M, Benotto M, et al. Development of a new aerosol monitoring system and its application in Fukushima nuclear accident related aerosol radioactivity measurement at the CTBT radionuclide station in Sidney of Canada. J Environ Radioact, 2011, 102: 1065-1069

4 Manolopoulou M, Vagena E, Stoulos S, et al. Radioiodine and radiocesium in Thessaloniki, Northern Greece due to the Fukushima nuclear accident. J Environ Radioact, 2011, 102: 796-797

5 Pittauerová D, Hettwig B, Fischer H W. Fukushima fallout in Northwest German environmental media. J Environ Radioact, 2011, 102: $877-880$

6 Masson O, Baeza A, Bieringer J, et al. Tracking of airborne radionuclides from the damaged fukushima Dai-ichi nuclear reactors by european networks. Environ Sci Tech, 2011, 45: 7670-7677

7 Bolsunovsky A, Dementyev D. Evidence of the radioactive fallout in the center of Asia (Russia) following the Fukushima Nuclear Accident. J Environ Radioact, 2011, 102: 1062-1064

8 Kim C, Byun J, Chae J, et al. Radiological impact in Korea following the Fukushima nuclear accident. J Environ Radioact, 2012, 111: 70-82

9 Long N Q, Truong Y, Hien P D, et al. Atmospheric radionuclides from the Fukushima Dai-ichi nuclear reactor accident observed in Vietnam. J Environ Radioact, 2012, 111: 53-58

10 Bowyer T W, Biegalski S R, Cooper M, et al. Elevated radioxenon detected remotely following the Fukushima nuclear accident. J Environ Radioact, 2011, 102: 681-687

11 Medici F. The IMS radionuclide network of the CTBT. Radiat Phys Chem, 2001, 61: 689-690

12 Chino M, Hiromasa N, Haruyasu N, et al. Preliminary estimation of release amounts of ${ }^{131} \mathrm{I}$ and ${ }^{137} \mathrm{Cs}$ accidentally discharged from the Fukushima Daiichi nuclear power plant into the atmosphere. J Nucl Sci Tech, 2011, 48: 1129-1134

13 Stohl A, Seibert P, Wotawa G, et al. Xenon-133 and caesium-137 releases into the atmosphere from the Fukushima Dai-ichi nuclear power plant: Determination of the source term, atmospheric dispersion, and deposition. Atmos Chem Phys Discuss, 2011, 11: 28319-28394

14 乔方利, 王关锁, 赵伟, 等. 2011 年 3 月日本福岛核泄漏物质输运扩散路径的情景模拟和预测. 科学通报, 2011, 56: 964-971

15 王海洋, 王晓霞, 黄树明, 等. 福岛第一核电厂事故源项估算及方法比较. 核安全, 2011, 2: 14-19

16 周程, 张起虹, 蒋云平, 等. 日本核泄漏期间南京地区空气中放射性气溶胶的监测和评价. 环境监测管理与技术, 2011, 23: 43-45

17 Preparatory Commission for the Comprehensive Nuclear-Test-Ban Treaty Organization. Certification of IMS radionuclide stations (with Guidelines for station installation). 1999

18 张利兴, 朱凤蓉. 核试验放射性核素监测核查技术. 北京：国防工业出版社, 2006. 72-74, 117

19 常印忠, 刘龙波, 王世联, 等. PMS-800 大流量大气颗粒物采样器的研制. 辐射防护, 2011, 31: 39-42

20 吴艳敏, 王旭辉, 刘龙波, 等. 高流速下气溶胶取样滤材的性能测试. 辐射防护, 2009, 29: 13-17

21 吴治华, 赵国庆，陆福全，等. 原子核物理实验方法. 第三版. 北京：原子能出版社, 1997. 272

22 常印忠, 王旭辉, 王世联, 等. 西安地区大气环境气溶胶样品中放射性核素监测. 核技术, 2008, 31: 796-800

23 张子斌, GOETZ A，韦冠一. 准确分析微量惰性气体的轻便四极杆质谱系统. 质谱学报, 2004, 25: S203-S204

24 中华人民共和国国家质量监督检验检疫总局. 电离辐射防护与辐射源安全基本标准. GB18871-2002. 2002

25 Nuclear Energy Agency, the Organisation for Economic Co-operation and Development. Chernobyl: Assessment of Radiological and Health Impacts-2002 update of Cherobyl: Ten Years on. Paris: OECD, 2002

26 庞巨丰, 郑桂芳, 王亮, 等. 切尔诺贝利事故对西安地区环境的影响和卫生学评价. 核技术, 1989, 12: 713-719

27 Biegalski S R, Bowyer T W, Eslinger P W, et al. Analysis of data from sensitive U.S. monitoring stations for the Fukushima Dai-ichi nuclear reactor accident. J Environ Radioact, 2011, 114: 15-21

28 Lozano R L, Hernández-Ceballos M A, Adame J A, et al. Radioactive impact of Fukushima accident on the Iberian Peninsula: Evolution and plume previous pathway. Environ Int, 2011, 37: 1259-1264

29 广飞虹，王世联，刘龙波，等。日本福岛核电站事故泄漏放射性核素漂移扩散状况分析. 原子能科学技术, 2012, 46: 1533-1536 